\title{
A Multi-Paradigm Approach to Grounded Theory
}

\author{
Walter Fernández
}

School of Accounting and Business Information Systems,

The Australian National University

email: walter.fernandez@anu.edu.au

Michael A. Martin

School of Finance and Applied Statistics,

The Australian National University

email: michael.martin@anu.edu.au

\section{Shirley Gregor}

School of Accounting and Business Information Systems, The Australian National University

email: shirley.gregor@anu.edu.au

Steven E. Stern

School of Finance and Applied Statistics,

The Australian National University

email: steven.stern@anu.edu.au

Michael Vitale

Melbourne Business School, University of Melbourne email: m.vitale@mbs.edu

\begin{abstract}
While grounded theory methodology was intended to be used as a general research method accepting any type of data, most grounded theory studies in the literature refer to research based on qualitative data. This paper aims to address this perceived neglect by describing our experiences and our approach while using grounded theory across qualitative and quantitative research paradigms. The case-based discussion presented in this paper, explains how the combination of these paradigms in exploratory studies can benefit research outcomes. The discussion follows the authors' approach to a competitive research grant opportunity that resulted in a comprehensive study into the use and management of information and communication technology in
\end{abstract}


Australian organisations. We propose that the use of classic grounded theory as a general research method enables researchers to capitalise on greater opportunities to participate in substantial team-based exploratory research endeavours.

\section{Introduction}

When the seminal work of Glaser and Strauss was published in 1967, grounded theory was proposed as a general method independent of a particular research paradigm. This early premise rarely, if ever, is mentioned in the current literature and thus many researchers perceive the method as being entirely within the domain of qualitative research, neglecting the fact that one of the cornerstones of the grounded theory method was the quantitative work of Barney Glaser (1964).

Glaser (1964), in an exploratory study of the professional careers of organisational scientists, presented many of the core elements of the grounded theory methodology. His study's goal was 'to explore for and to develop generalised formulations on these careers'. Thus, the research was not about testing preconceived theoretical propositions but rather its purpose was to develop plausible relations between variables that could 'guide sociologists of science - as well as of occupations, professions, and organisations'.

Moreover, Glaser (1964) was able to acknowledge and include two important aspects of studies of sociological processes, namely: that a process exists within multiple contextual conditions and that these contexts are subject to variations as they present several stages or phases. The study described a process in conceptual terms in such a way that variations of context were accounted for in the theoretical formulation emerging from the research. Given this recognition of contextual issues, usually associated with qualitative studies, it may be surprising to some that for his foundational study, Glaser used secondary quantitative data and extant literature as data sources. By using extensive secondary quantitative data to search for patterns and the literature to inform the research, Glaser was able to detect and enrich many emergent concepts.

The exploratory analysis of quantitative data enabled the identification of processes and discovery of properties of these processes. For example, the property of 'integration' in a process of facing career concerns describes the concept of individuals choosing to integrate with peers according to their common circle of concern. By integrating with different members of the circle, the scientists were able to solve their own career concerns and to move forward in their careers. Another example can be found in the process of career advancement, where recognition was a property that resolved scientists' career concerns. 
The second book presenting the results of grounded theory research was Awareness of Dying (Glaser and Strauss, 1965), which was an exploration of the social process of dying in American hospitals. The overall research approach taken in this study was, in many ways, similar to Glaser (1964) and yet, as Glaser and Strauss conducted intensive fieldwork based on interviews and observations, the data was qualitative in nature. It was this work on dying that consolidated the grounded theory method and gave it its initial recognition.

Glaser and Strauss (1967) clearly state that grounded theory is a general method that accepts both qualitative and quantitative data, and claimed that 'grounded theory is a general method of comparative analysis' (emphasis in original). Indeed, Glaser and Strauss (1967) described the 'slices of data' collected for theoretical sampling as varied, providing researchers with limitless options for data gathering including different collection techniques, data types and ways of analysing the data with the objective of generating 'different views or vantage points from which to understand a category and to develop its properties'. In other words, the nature of the data is not important in itself. More significant is the role the data play in providing evidence for useful conceptualisations.

Regardless of early descriptions and evidence, it is difficult to find recent examples of grounded theory studies that have used quantitative data or mixed data approaches. This is clearly the case in information systems (IS) research, a late adopter of the grounded theory method and where, to our knowledge, all published grounded theory studies are of a qualitative nature.

While this situation is neither desirable nor undesirable per se, we suggest that by failing to perceive grounded theory as a general research method, IS grounded theorists could be missing opportunities to participate in important collaborative research endeavours. In IS research it is often necessary to combine qualitative and quantitative research skills to analyse complex socio-technical phenomena and to satisfy the needs of diverse stakeholders. Furthermore, in IS it is important to produce empirical studies that are both academically rigorous and relevant to the information and communication technology (ICT) industry (Benbasat and Zmud, 1999) and grounded theory can contribute to rigorous and relevant research outcomes (Fernández and Lehmann, 2005).

This paper aims to address the apparent neglect of the general nature of the grounded theory method by describing the case of a recent study that used both qualitative and quantitative data. In the study we are about to describe, a team of researchers from different backgrounds decided to adopt key premises from grounded theory methodology for their exploratory investigation; in particular, the detection of patterns and the desire to discover what is going on in a particular substantive field. The ability to use a mixed-data approach was also a differentiation strategy in competing for the research opportunity. 


\section{The case of the Australian ICT study}

In early 2004, the Australian Government's Department of Communication, Information Technology and the Arts (DCITA) called for a competitive tender to commission a study entitled 'ICT, organisation and management - the strategic management of technology'. DCITA wanted to 'examine the relationship between ICT, organisation and management and the way in which it contributes to productivity, organisational transformation and establishing sustainable competitive advantage' ${ }^{1}$

To achieve the research objective, DCITA requested that the study:

[conduct] a comprehensive survey, examining the competitive and business settings under which ICT is implemented by Australian enterprises;

examine the interaction between ICT and organisational and management strategies and processes such as change management, business process review, strategy alignment, organisational restructuring and competency development;

contribute to understanding and knowledge of the interdependence between social and economic processes and ICT in enterprises and the factors that influence the adoption of particular management and organisational strategies; and

report on the outcomes of this research in a form that also provides practical advice to firms, industry and governments on the principles and practices that contribute to successful ICT implementation and management. $^{2}$

DCITA was open to different approaches and, in order to successfully compete, our team needed to produce a convincing conceptual framework and a methodology that could enable the attainment of DCITA's stated research objectives. The research strategy is explained next.

\section{The proposed research framework}

Our brief was to examine the circumstances and settings in which ICT is implemented by Australian organisations in a number of industry contexts. In particular, DCITA was interested to explore the relationships between ICT, the environment, the industry, and organisation and management, and the associated contribution of ICT to business value.

As we had only a few months to collect and analyse considerable amounts of data, we needed to use an efficient method. Simultaneously, our methodology

1 DCITA's Tender Brief No. NCON/04/10 p.1

2 DCITA's Tender Brief No. NCON/04/10 p.2 
needed to fulfil DCITA's dual requirements for rigour and relevance and the study had to withstand academic scrutiny as well as provide meaningful lessons and outcomes for the Australian ICT industry.

To compete for the grant, we adopted an integrated multi-paradigm research strategy that involved different types of explorations, building on each other and on previous empirical research. By systematically adding layers of knowledge to our understanding of the problem domain, we could aim to go as far as possible given the time constraints and yet produce rigorous and useful research. To fulfil the requirements of the request for tender, we believed it was critical to follow an efficient theory construction approach in which research actions produced slices of knowledge that can be aggregated and explored in more detail by successive research actions.

Our basic objective was to use statistical methods to discover patterns (also called data mining) as well as interviews and focus groups to obtain rich data upon which we could ground our enquiry before the survey and then further develop and explain the patterns emerging from the survey. The design and execution of these activities took advantage of the diverse research skills within the team. We had six people working in the project: three IS experts (one skilled in the use of grounded theory), two statisticians and one project manager/industry consultant.

The study was designed to have three phases, each including data collection and analysis: Phase One in which the survey instrument was developed, Phase Two in which the survey was conducted and analysed, and Phase Three, which involved the conduct and analysis of interviews and the final integration of conceptualisations, as discussed in the following sections.

\section{Phase One: survey instrument development}

Critical to our objectives was the development of a survey instrument based on empirical theory from prior research work, and grounded in the current context of the Australian industry. This grounding activity was essential to ensure a sound basis for identification and specification of constructs, rigour in the project methodology and to give legitimacy and credibility to the results. The flow of main tasks and outputs of this phase are represented in Figure 1, which is explained in more detail below. 


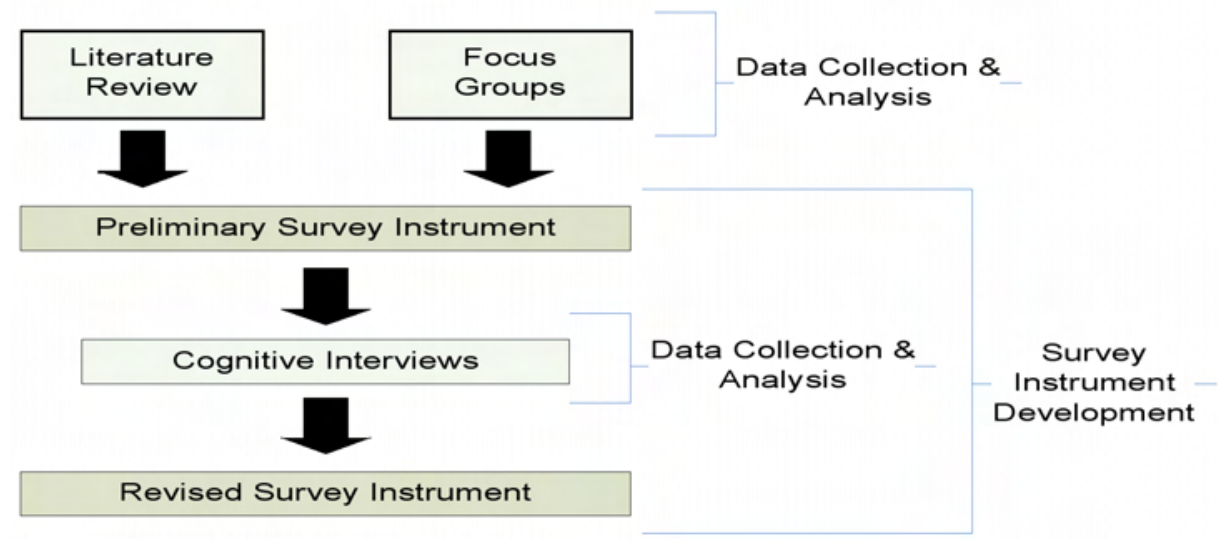

\section{Figure 1: Activities and outcomes - Phase One.}

We investigated the literature across empirical studies conducted worldwide. The aim was to find well-tested survey models, although as a starting point only since uncritically relying on those models could ignore peculiarities of the Australian context. In parallel with the literature review, we convened a series of focus groups with business executives and senior ICT managers.

The focus groups were central to the initial development of the survey instrument. A total of 27 organisations took part in four focus groups held in Melbourne, Sydney and Canberra. The results were used to ensure that the survey instrument was grounded in, and relevant to, the current circumstances and settings of Australian organisations across a range of industries, organisation sizes, and geographical locations.

The focus groups used the Nominal Group Technique (Delbecq et al., 1986) to develop and prioritise a list of issues associated with the successful use of ICT. This technique allows the participants to discuss and prioritise their own issues rather than forcing a prioritisation based on extant literature. The experience of the focus group participants regarding the benefits arising from ICT aligned well with previous empirical research that categorised benefits as being strategic, informational and transactional. Factors identified as important to successful ICT use included: strategic planning, education and training, cost, support for ICT, effective client-supplier relationships, business case understanding, and effective management decision making. These key observations from the focus groups were used as inputs to the development of a draft survey instrument.

To help reduce the ambiguity often present in questions, the draft survey instrument was then refined through seven face-to-face cognitive interviews conducted in Sydney and Canberra. Participants were asked to report on how they would arrive at their answer to a particular question rather than on what 
their answer would be. For example, what meaning did they ascribe to the terms used and what context did they apply when considering their answer.

Based on the results from these interviews, we identified many problems that respondents had in understanding questions, and revised the survey questions accordingly. Finally, the survey instrument was vetted and cleared by the Australian Bureau of Statistics (ABS) Statistical Clearance House, a mandatory step for government-sponsored research in Australia.

\section{Phase Two: conducting and analysing the survey}

The aim of Phase Two was to collect and analyse the data, searching for important concepts and their attributes. The flow of main tasks and outputs of this phase are represented in Figure 2, which is explained below.

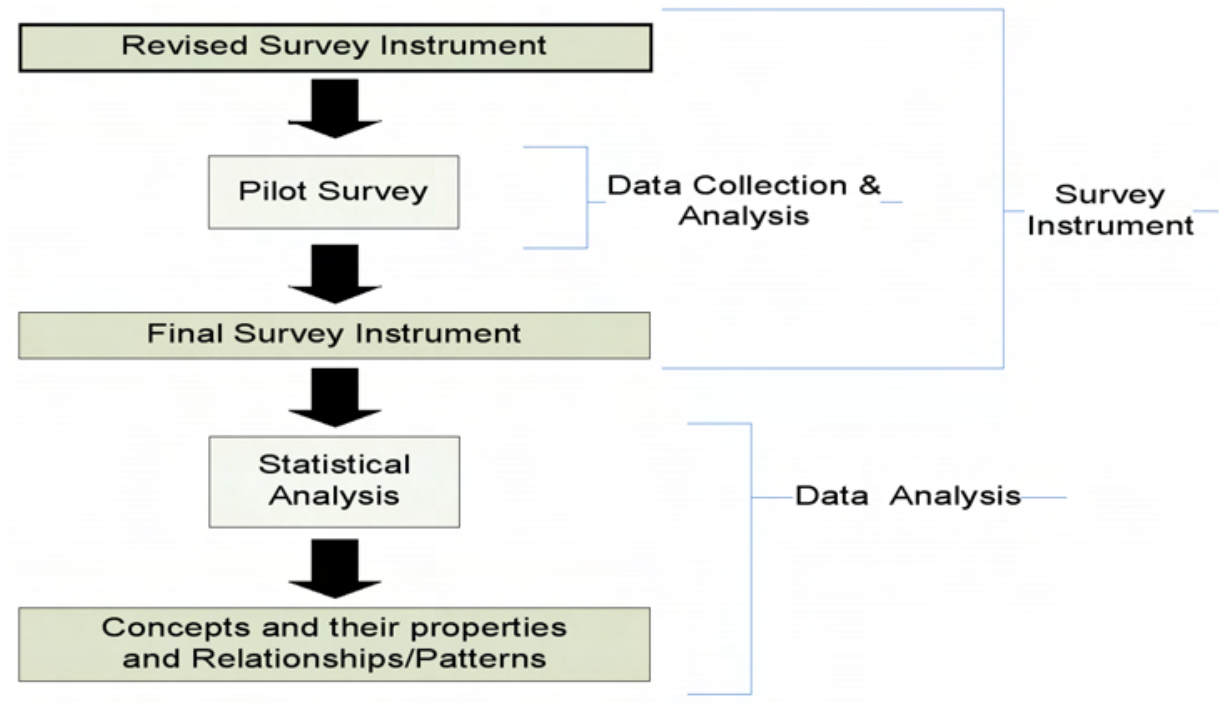

Figure 2: Activities and outcomes - Phase Two.

The data consisted of observations of a number of variables for each of the 1050 organisations surveyed. The sampling frame was drawn from the Dun and Bradstreet database of Australian organisations. The sampling methodology used stratification by business size, so that the final sample included 225 large organisations (100 or more employees), 450 medium organisations (20-99 employees) and 375 small organisations (5-19 employees).

The approach was also intended to cover 15 of the Australian New Zealand Standard Industry Classification (ANZSIC) codes, so initially quotas by industry as well as size were planned. While the allocated sample size was not met for all industries, the number of responses obtained within each of the 15 industries was sufficient for further analysis. The initial sampling frame included 5380 organisations. Advance letters were sent to 2600 of these organisations to increase 
response rates and $63 \%$ of these companies were also contacted by telephone. The response rate was $31 \%$. Details of the actual numbers of responses obtained by industry as well as organisation size are provided in Table 1.

\begin{tabular}{llll}
\hline \multirow{2}{*}{ Industry } & \multicolumn{3}{l}{ Number of Responses } \\
\cline { 2 - 4 } & Small Size & Medium Size & Large Size \\
\hline Agriculture, Forestry and Fishing & 31 & 37 & 15 \\
\hline Manufacturing & 33 & 35 & 17 \\
\hline Electricity, Gas and Water Supply & 12 & 12 & 18 \\
\hline Construction & 27 & 39 & 12 \\
\hline Wholesale Trade & 37 & 30 & 9 \\
\hline Retail Trade & 39 & 32 & 13 \\
\hline Accomm, Cafes and Restaurants & 25 & 15 & 8 \\
\hline Transport and Storage & 19 & 29 & 7 \\
\hline Communication Services & 33 & 35 & 16 \\
\hline Finance and Insurance & 13 & 31 & 11 \\
\hline Property and Business Services & 42 & 32 & 20 \\
\hline Govt Administration and Defence & 5 & 27 & 22 \\
\hline Education & 11 & 25 & 17 \\
\hline Health and Community Services & 26 & 36 & 18 \\
\hline Cultural and Recreational Service & 22 & 35 & 225 \\
\hline Total & 375 & 450 & \\
\hline
\end{tabular}

Table 1: Sample sizes.

The survey was telephone-based since this technique enabled survey data from a large number of Australian organisations to be gathered within the required time and cost constraints. Also, to alleviate the problem of the time constraint, we contracted a quality-accredited research organisation, here called Social Research Group (SRG), to collect data for the research team using a computer assisted telephone interviewing (CATI) facility. After the first 20 interviews, which were used as a pilot, all fieldwork was completed in time, between 8 June 2004 and 7 July 2004, and we were ready for the data analysis stage.

The purpose of our data analysis was to discover patterns and to detect significant structure in the data. Many modern statistical procedures begin with the simple purpose of detecting significant structure in high-dimensional data; that is, where the dimension of the data is in the hundreds of covariates. The term 'significant structure' has many interpretations, the two most useful of which state that a significant structure exists when:

- the data suggest that there is a relationship between a particular variable (variously termed a response or dependent variable) and a subset of the other variables (called predictors or independent variables), and the data suggests the nature of that relationship; and

- the dimension of the covariate space can be reduced by discovering relationships among the predictors. That is, the original high-dimensional 
covariate space has a meaningful lower-dimensional subspace that possesses most of the important properties of the original space.

These interpretations reflect the broad principle of parsimony. That is, they attempt to answer the question 'can the information carried in a high-dimensional data set be adequately understood in a low-dimensional analogue?' and, further, to explore and interpret relevant relationships within the resultant low-dimensional space.

Structure of the first kind has classically been captured by parametric regression models, which seek to represent the structure in terms of straightforward linear models relating response and covariates. The strength of such models is that they are very compact, low-dimensional representations of the data, but their main drawback is that in cases where the underlying structure is not simple or linear they can fundamentally fail to capture what is really going on in the data. A broad overview of statistical modelling techniques for discovering structure in data of the type we describe in this paper is given in an excellent monograph entitled The Elements of Statistical Learning (Hastie et al, 2001). This book describes basic parametric techniques and discusses in detail the strengths and weaknesses of the classical parametric approach to model fitting.

More modern, non-parametric techniques relax classical linearity and distributional assumptions, thereby allowing more flexible and realistic models, which are inherently data-based. Examples of such methods include:

- generalised linear models (Nelder and Wedderburn, 1972; McCullagh and Nelder, 1989) that allow for non-normal error assumptions and certain types of non-linear relationships;

- generalised additive models (Hastie and Tibshirani, 1986) that seek to find the best fitting additive relationship between transformed versions of the response and covariates; and

- regression trees (Breiman et al., 1983) that recursively partition the high dimensional space into homogeneous subspaces using simple binary decision rules at each step.

The key advantage of these techniques is their flexibility - they essentially trade off extremely compact descriptions of the response surface (such as simple lines or planes) against better fitting but more complex models.

In our analysis, the response variable - that is, the construct we wished to model in terms of other properties of the businesses involved - was not initially precisely defined as the outcome to a single survey question but was rather summarised in survey responses to 22 interview questions. In other words, the response space was initially a 22-dimensional space, and the first goal of the analysis was to isolate a smaller space spanned by interpretable variables that 
could be used as response variables in our model, and which adequately captured the important properties of the original 22-dimensional response space.

We employed a classical multivariate technique, factor analysis, to discover meaningful structure amongst covariates, and to provide low-dimensional analogues to high-dimensional covariate spaces.

Factor analysis seeks to account for variability in the original high-dimensional space by identifying relatively few common, underlying factors (linear combinations of the original covariates) that explain similar variability to that possessed by the original high-dimensional space. Further, factor analysis attempts to assign simple meanings to the resultant factors based on the characteristics of their constituent variables. Factor analysis specifically posits the existence of a small number of common factors that account for a reasonable proportion of the variability in the higher-dimensional space up to a set of 'unique' elements that cannot be accounted for by the small set of common factors. Factor analysis is described in detail in many texts on multivariate statistical methods - see, for example, Lattin et al. (2003).

We carried out a factor analysis in an attempt to reduce the complex 22-question response into a form that was both lower-dimensional and easily interpretable in terms of constructs isolated in the foregoing qualitative analysis. A four-factor solution isolated four broad types of value arising from the use of ICT: informational (i.e. increasing the quality, quantity and availability of valuable information), strategic (i.e. creating a competitive advantage), transactional (i.e. leading to efficiencies) and transformational (i.e. enabling organisational change).

The four-factor solution allows a compact representation of the complex response space in terms of easily interpreted factors, the features of which arose from the earlier, qualitative phase of our investigation. These four factors together explained over $60 \%$ of the variation present in the original 22-dimensional response space, and so this solution allowed for both significant dimension reduction as well as a confirmation of existing notions of what sorts of benefits might arise from ICT implementation.

\section{Phase Three: interviews and reporting}

The third and final phase of the project was designed to expand and explain concepts emerging from the previous phases and to integrate these concepts into a report. To achieve this aim, we used both quantitative and qualitative techniques. Figure 3 represents the activities of this phase, which are explained in more detail below. 


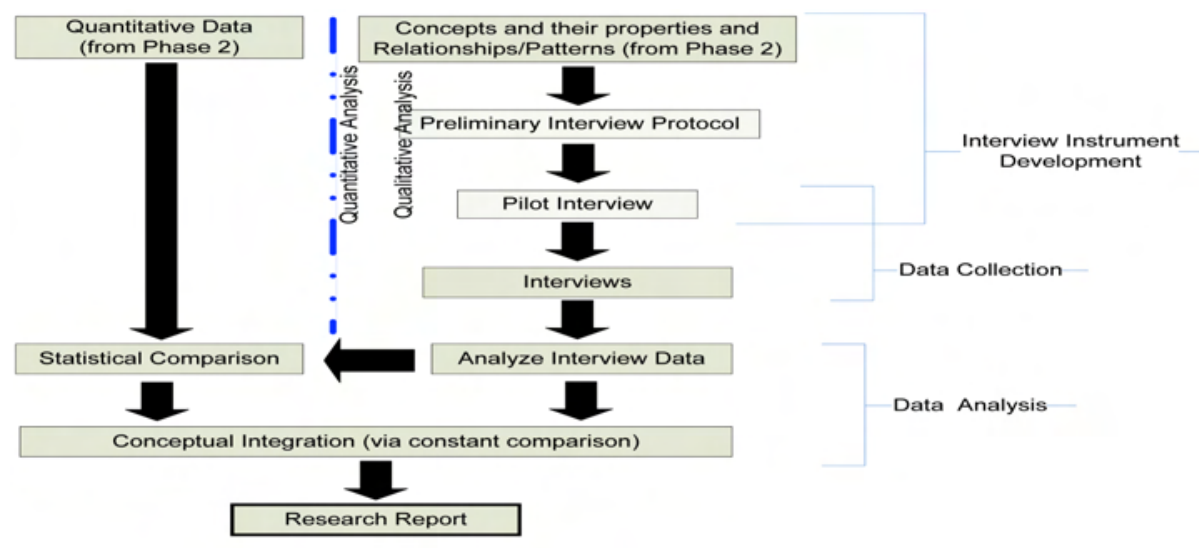

Figure 3: Activities and outcomes - Phase Three.

As described above, Phase One was mainly qualitative and Phase Two was mainly quantitative. In Phase Three, however, this distinction becomes to a certain extent meaningless. Once the analysis of our interview data started to result in concept emergence, we were engaged in an almost simultaneous process of comparing ideas and concepts emerging from the interviews with the quantitative data from the survey. Through the constant comparison of these concepts against the raw data collected in Phase Two, we were able to test the conceptual validity of what we considered important. Nevertheless, to explain how we conducted our work, the next two subsections describe the qualitative and quantitative components separately.

\section{Qualitative component}

Fifty structured interviews were conducted between the 2nd and 23rd July 2004. Participants were recruited by SRG by contacting those who indicated during the large-scale survey stage that they would be willing to participate in further research for this project. The conduct of the interviews was also outsourced to SRG. The majority of these interviews were performed face-to-face, others by telephone. The purpose of the interviews was to collect data regarding specific contextual and managerial aspects related to ICT use and implementations considered as significant by the survey participants. The interviews were designed to collect descriptions of:

- How the use and implementation of ICT impacted the organisation;

- How the organisation managed these uses and implementations;

- Results achieved from using and implementing ICT;

- Contribution of ICT to overall organisational change; and

- Current organisational challenges and the role of ICT in facing these challenges. 
An interview protocol was developed to ensure a consistent approach was taken to the interviews. We conducted four pilot interviews to fine-tune the questions before conducting the remaining 46 interviews. The final protocol allowed different interviewers to conduct the interviews in different Australian states, in similar fashion and using the same questions. Yet the questions were open, allowing the participants to tell their own stories and thus enabling us to collect rich information. The interviewers took comprehensive notes, which were manually coded using ATLAS.ti, a software tool for text analysis. The analysis of interview notes resulted in 301 pages of quotations.

The initial aim of the analysis was to produce a set of quotations that could be used to illustrate the findings from the extensive survey previously conducted. However, the interviews were extremely valuable as they revealed interesting examples supporting the findings of the telephone based survey and they also showed core patterns interrelating human and organisational behaviour with benefit realisation, in particular as follows:

- Being ICT aware. Awareness of ICT capabilities influenced the style of management adopted and also increased the likelihood of achieving benefits from organisational learning and organisational transformation.

- Being open to organisational transformation. Organisations that achieved the most significant benefits from ICT were able to exploit their new ICT capabilities to transform their business processes and to create new opportunities.

- Being persistent in benefit realisation. The process of benefit realisation is governed by time. This is so because successful use of ICT demands a continuous effort to learn and to change, causing a lag between implementing ICT and realising the full benefits of that implementation.

Our research indicated that ICT benefit realisation is mainly influenced by managerial choices and behaviours. We found a widespread realisation of value from ICT in which organisations with clear and strategic reasons for ICT investment (internal ICT impetus), good practice in ICT management and openness to organisational transformation and change were associated with the achievement of significant benefits. Contrary to expectations, we also found that size of firm, size of ICT investment, ICT applications adopted, ICT support type, size of in-house ICT team, ICT decision maker type and perceived inhibitors were not so important.

The analysis of the notes indicated a pattern in which being ICT savvy was perceived as a major factor in benefiting from ICT implementations. This pattern was interesting because it was not related to the size of the companies; rather, it appeared across our sample of organisations. Furthermore, the pattern took the shape of self-fulfilling prophecies or virtuous/vicious circles as used by Weick (1979) to explain the effect of multiple causal loops on the fate of the 
system. Contrary to other studies, our data was showing that one of the most important factors influencing benefit realisation from ICT in Australia was related to human will and attitudes towards technology. To further explore this pattern, we used a number of statistical techniques to search for significant relationships relating business value to other business features.

\section{Quantitative component}

Having isolated a low-dimensional response, the next step in the analysis was to relate those responses to the other business features through an explicit modelling step designed to isolate which business features were most associated with high business value being achieved. Simple linear regression, though compact and easy to understand, was not a reasonable approach in this case because of the lack of a clear linear relationship between response and covariates. Rather, a non-parametric method, regression tree modelling, was used to find significant relationships between the response(s) and the predictors.

Tree methods use an approach called recursive partitioning to model the high-dimensional relationship in terms of local features of the data. The basic premise of regression tree modelling is to seek binary splits in the data that yield large differences in the response, and then to recursively split on subsequent variables to find finer structure. A reasonable analogy that may be helpful in visualising tree modelling is that of representing a smooth surface using building blocks; locally, the blocks are flat, but if different-sized blocks are used and sufficiently many blocks employed, a smooth surface can be approximated reasonably well. Such techniques are most effective when simple linear models fail to adequately capture the relationships in the data. These techniques are also very flexible since they can adapt to 'shapes' in the relationship by splitting repeatedly on a small set of variables, revealing complex interactive effects that simple linear models can fail to capture. Classification and regression trees (CART) were developed by Breiman et al (1983) as a non-parametric alternative to traditional parametric classification and regression procedures. In the current context, regression, rather than classification, trees were used in our analysis. The use and broad features of regression tree models are discussed in detail by Hastie et al (2001).

The results of our analyses revealed that the covariates that most reliably predicted high business value arising from ICT implementation were related to ICT awareness in businesses along with the persistence with which businesses pursued their ICT strategies. The results of our tree models repeatedly showed that these variables were those on which the tree preferred to split, with other 'demographic' variables such as business size and so on only appearing fairly low in the tree structure. This statistical procedure was conducted to discover how business benefits were achieved, and was carried out in parallel with the qualitative activities described above. 
Critically, the results of our quantitative analysis independently affirmed our main qualitative findings while also helping to refine our understanding of how concepts such as 'ICT awareness' might be measured. Further, the constant comparison between incidents and the further grounding of emerging concepts in quantitative data was a powerful feature of our research approach.

\section{Conclusion}

We have described a research case and the main actions taken to fulfil the research objectives and, to conclude, we present some thoughts regarding what we did and the lessons we learned.

The interviews were extremely valuable in allowing us to detect patterns and move beyond the limited goal of simple description. That is, while the data gathered from the interviews provided some interesting examples to support the findings of the telephone based survey, it also showed core patterns relating human and organisational behaviour with benefit realisation. These patterns were important because they allowed us to engage in further exploration of the quantitative data through the use of sophisticated data mining statistical techniques.

Going beyond description is one of the most important and powerful aspects of grounded theory. We feel that while more could have been achieved in this regard, we were able to detect important concepts and their interrelationships in such a way that it was possible to present a theory. For example, the comparison of concepts across qualitative and quantitative data resulted in the identification of three core concepts that we used to explain the relationship between ICT, organisation and management and the way in which it contributes to productivity, organisational transformation and establishing sustainable competitive advantage (Gregor et al., 2005).

The study had a number of limitations. Non-response bias may have influenced the results. However, we have no direct evidence of non-response bias and the use of industry best practice standards by SRG in conducting the survey assisted in reducing the non-response bias to the minimum possible in such circumstances. Yet, the patterns observed 'worked' in the studied substantive field, and concerns regarding non-response bias are arguable from the classic grounded theory perspective since the unexpressed concerns of indifferent or unsuccessful managers would only add another dimension to the study rather than negate the validity of the findings in the studied substantive field.

Another issue with this study relates to its depth. This was accepted as a limitation of scope and time imposed by the organisation that commissioned the study. However, by adopting a grounded theory approach we were able to produce meaningful results in a short period of time and also to provide the foundations for a second study to extend the theory. 
Before the study, the team thought that a diverse research team could work together cohesively by following a grounded theory approach. However, it was through our research actions that we found answers to questions such as:

- Is it possible for grounded theory techniques and principles to contribute to other approaches?

- Are these principles and techniques flexible enough?

- Is this approach efficient enough to meet the deadline?

- Is the approach effective enough to meet expectations of relevance?

We now believe that the answer to these questions is yes. In doing the research we gained understanding of our team strengths and complementary skills, discovered key synergies between our qualitative and quantitative team components, and developed further research capabilities. This approach can work successfully for us; it enhances our team's capabilities to both gain access to grants and to produce research that is rigorous and relevant to our target community.

\section{References}

Benbasat, I. and Zmud, R. W. 1999, MIS Quarterly, vol. 23, pp. 3-16.

Breiman, L., Friedman, J. H., Olshen, R. A., and Stone, C. J. 1983, CART: Classification and Regression Trees. Wadsworth: Belmont, CA.

Delbecq, A., Van de Ven, A. H. and Gustafson, D. 1986, Group Techniques for Program Planning: A Guide to Nominal Group and Delphi Processes., Green Briar Press, Middleton, Wisconsin, USA.

Fernández, W. D. and Lehmann, H. P. 2005, The grounded theory review: An international journal, vol. 5, pp. 79-107.

Glaser, B. G. 1964, Organisational Scientists: Their Professional Careers, The Bobbs-Merrill Company, Inc., Indianapolis.

Glaser, B. G. and Strauss, A. L. 1965, Awareness of Dying, Aldine Publishing Company, New York.

Glaser, B. G. and Strauss, A. L. 1967, The Discovery of Grounded Theory: Strategies for Qualitative Research, Aldine Publishing Company, New York.

Gregor, S. D., Fernández, W. D., Holtham, D., Martin, M. A., Stern, S. E. and Vitale, M. R. 2005, Achieving Value from ICT: Key Management Strategies, Department of Communications, Information Technology and the Arts, Canberra.

Hastie, T. and Tibshirani, R. 1986, 'Generalised additive models', Statistical Science, vol. 1, pp. 297-318. 
Hastie, T., Tibshirani, R. and Friedman, J. 2001, The Elements of Statistical Learning, Springer-Verlag, New York.

Lattin, J., Carroll, D. and Green, P. E. 2003, Analysing Multivariate Data, Duxbury Press, Belmont, CA.

McCullagh, P. and Nelder, J. 1989, Generalised Linear Models, Chapman and Hall, London.

Nelder, J. A. and Wedderburn, R. W. M. 1972, 'Generalised linear models', Journal of the Royal Statistical Society, Series A (General), vol. 135, no. 3, pp. 370-84.

Weick, K. E. 1979, The Social Psychology of Organising, Addison-Wesley, Reading, Mass. 